\title{
Carcinoembryonic Antigen: A Predictor of Inflammatory Condition in Patients with Allergic Bronchopulmonary Aspergillosis?
}

Huan Ge

Xiangya Hospital Central south university

Feifei Yin

Xiangya Hospital Central south University

Yuanyuan Jiang

Xiangya Hospital Central South University

Lisha Luo

Xiangya Hospital Central South University

Shuanglinzi Deng

Xiangya Hospital Central South University

Yingyu Zhang

Xiangya Hospital Central South University

Daimo Zhang

Xiangya Hospital Central South University

Yifei Yang

Xiangya Hospital Central South University

Huan Tang

Xiangya Hospital Central South University

\section{Runjin Cai}

Xiangya Hospital Central South University

Xinyue Hu

Xiangya Hospital Central South University

Chendong Wu

Xiangya Hospital Central South University

Xiaozhao Li

Xiangya Hospital Central South University

Juntao Feng ( $\nabla$ jtfeng1976@csu.edu.cn )

Xiangya Hospital Central South University https://orcid.org/0000-0002-3945-1576 
Keywords: Allergic bronchopulmonary aspergillosis (ABPA), Carcinoembryonic antigen (CEA), Asthma.

Posted Date: January 15th, 2021

DOI: https://doi.org/10.21203/rs.3.rs-145782/v1

License: (c) (1) This work is licensed under a Creative Commons Attribution 4.0 International License. Read Full License 


\section{Abstract}

Background: Allergic bronchopulmonary aspergillus (ABPA) is a complex non-infectious pulmonary benign disease caused by immune response against to aspergillus. Carcinoembryonic antigen (CEA) is a tumor marker but also elevated in some benign diseases. Few studies on ABPA with elevated serum CEA levels have been reported.

Objects: This study aims to comb the clinical characters of ABPA with elevated serum CEA. Methods: 20 patients diagnosed as ABPA were divided into two groups (CEA normal and CEA elevated) for retrospective analysis. The eosinophil count and ratio, IgE level in the pretherapy and post-treatment were compared. Serum samples of patients with ABPA $(n=20)$ and asthma $(n=20)$, healthy controls $(n=20)$ were collected. Levels of cytokines (IL-4, IL-5, GM-CSF, IFN-Y) were analyzed by enzyme-linked immunosorbent assay.

Results: We found that in ABPA patients with normal serum CEA levels, eosinophil counts and IgE levels decreased more obviously after treatment. Besides, we established higher serum levels of IL-4, IL-5, GMCSF and IFN-Y in ABPA patients with elevated serum CEA levels.

Conclusion: For the ABPA patients with elevated serum CEA levels, CEA may serve as a monitoring indicator for severity and treatment efficacy of ABPA.

\section{Introduction}

Allergic bronchopulmonary aspergillus(ABPA) is a complicated disorder due to immune response to aspergillus adhering to the airway, characterized by central tracheal dilatation and repeated pulmonary infiltration ${ }^{1}$. The classic pathological characteristics of ABPA include mucoid impaction, eosinophilic pneumonia, bronchiolitis obliterans, granulomatous bronchiolitis and pulmonary fibrosis ${ }^{2,3}$. The patients with ABPA often present with fever, wheezing, cough, bronchial hyperreactivity, or hemoptysis ${ }^{4}$. Human studies and animal experiments have shown that Th2 cells and relevant cytokines play a critical role in the progression of $\mathrm{ABPA}^{5}$. It is well known that CEA is a broad-spectrum tumor marker, which usually elevated in a wild variety of tumors such as colorectal cancer, breast cancer and lung cancer ${ }^{6}$. An increased levels of CEA can be acquired by a blood test, which commonly used for diagnosis, recurrence or progression of malignant diseases ${ }^{7}$. Some benign diseases such as viral hepatitis, cirrhosis, pancreatitis and ulcerative colitis have also reported elevated serum CEA levels ${ }^{8,9}$. Rarely, ABPA has been described with elevated CEA.

In this study, we observed 20 patients with ABPA, their total blood IgE levels, peripheral blood eosinophil counts and ratios before and after treatment and baseline CEA levels were collected, then evaluate the changes. Furthermore, we also detected a series of relevant cytokines in partial of the patients, compared with health volunteers and asthma patients. 


\section{Methods}

\section{Study subjects and patients:}

We retrospectively analyzed 20 patients diagnosed with ABPA from Xiangya Hospital of Central South University (each patient was only included once) from January 2017 to May 2020. The diagnosis of ABPA were in accordance with criteria of working group under the International Society for Human and Animal Mycology (ISHAM). Patients' data were collected including age, gender, blood routine, pulmonary function, imaging, bronchoscopy, IgE (total and specific), serum CEA, CRP, ESR and clinical and medication records. Table 1 demonstrates the details of the ABPA patient groups. The diagnosis of asthma was based on the Global Initiative for Asthma (GINA) guidelines. Patients with serious illness, malignancy or other diseases with elevated serum CEA were excluded. Twenty healthy volunteers were recruited into the healthy control group. Serum of 9 ABPA patients were collected prior to treatment. All asthma samples were collected in our outpatient clinic before treatment. Levels of IL-4, IL-5, IFN- $\gamma$ and GM-CSF in serum were analyzed by ELISA according to the manufacturer's instruction (All kits were purchased from eBioscience). 
Table 1

Demographics and Clinical Characteristics of 20 ABPA Patients

ABPA (20)

$P$ value

Serum CEA normal (14) Serum CEA elevated (6)

\section{Demographics}

Sex (Male / Female)

Age $($ mean $\pm S D$, range $)$

Clinical symptoms

Wheeze

Dyspnea

Chest tightness

Cough

Hemoptysis

\section{Pulmonary function test}

FVC (\% pred)

FEV1(\% pred)

FEV1/FVC ratio (\%)

Immunological characteristic

Af slgE positivity (\%)

Af slgE levels (kUA/L)

Bronchoscopy

Suppurative inflammation

Sputum jammed

\section{Computed tomography}

Central bronchiectasis

Nodules

Fleeting infiltration

Mucous plugs
$5 / 9$

$47.9 \pm 15.1(20-75)$

$21.5 \%$

$35.7 \%$

$7.0 \%$

$64.2 \%$

$21.4 \%$

76.10(52.90-109.00)

60.49(28.40-104.30)

72.88(33.14-90.76)

$100 \%$

10.25(0.42-29.8)

$100 \%$

$11 \%$

$100 \%$

$83.3 \%$
82.53(70.90-99.00)

0.17

64.78(50.40-91.50)

0.65

67.04(58.85-77.91)

0.50
1.0

0.66

$33.3 \%$

$33.3 \%$

$0.0 \%$

$100.0 \%$

$0.0 \%$
$100 \%$

10.87(0.52-42.5)

0.9
$64.3 \%$

$22 \%$

$85.7 \%$

$7 \%$
$100 \%$

$0 \%$

$100 \%$

$80 \%$

Abbreviations: ABPA, allergic bronchopulmonary aspergillosis; CEA, Carcinoembryonic antigen;FEV1, forced expiratory volume in $1 \mathrm{~s}$; FVC, forced vital capacity; 


\begin{tabular}{|c|c|c|c|}
\hline & \multicolumn{2}{|l|}{ ABPA (20) } & \multirow[t]{2}{*}{$P$ value } \\
\hline & Serum CEA normal (14) & Serum CEA elevated (6) & \\
\hline Treatments & $100 \%$ & $100 \%$ & - \\
\hline Use of oral glucocorticoids (\%) & $100 \%$ & $100 \%$ & \\
\hline Use of antifungal agents (\%) & & & \\
\hline
\end{tabular}

\section{Statistical analysis:}

Differences between two groups were analysed by student's tests or Mann-Whitney test. Comparisons between different groups were evaluated by the one-way ANOVA test, Kruskal-Wallis test were used when appropriate. The statistical analysis was performed with GRAPHPAD PRISM 8.0 software. The significance levels were set at $\mathrm{P}<0.05$.

\section{Results}

\section{Clinical materials}

As shown in Table 1, among 20 ABPA patients, 6 of them have significant elevated serum CEA levels above baseline and others were normal. Most patients clinically presented with wheeze, cough, dyspnea, chest tightness and hemoptysis. The lung function also indicated ventilatory dysfunction most. All the patients received glucocorticoid and antifungal treatments after diagnosed as ABPA. However, we found that most ABPA patients with elevated CEA presented with mucus plugs in bronchoscopy and manifestations of CT compare with ABPA patients with normal serum CEA. As we can see, The IgE levels, peripheral blood eosinophil counts and ratios did not differ in two groups. The erythrocyte sedimentation rate (ESR) and C-reactive protein (CRP) were also found no difference (Fig. 1). After a period of combination therapy of corticosteroid and antifungal agent treatments, the serum CEA levels were decrease than baseline levels. The levels of serum total IgE, peripheral blood eosinophil counts and ratios were both decreased in the two groups, while the degree of decline was more pronounces in the patients with normal serum CEA (Fig. 2).

\section{2.cytokine Levels}

We collected serum samples from the ABPA patients, asthma patients and healthy volunteers, and detected the concentrations of cytokines. We found that ABPA patients with elevated serum CEA produced more IL- 4 and IL- 5 than ABPA patients with normal serum CEA. In addition, production of IL-4 and IL-5 were significantly higher in ABPA patients with elevated serum CEA than in healthy subjects. Moreover, expression of IFN-Y and GM-CSF showed the similar trend in all groups (Fig. 3). 


\section{Discussion}

ABPA was first reported in 1952 by Hinson et $\mathrm{al}^{10}$. In the past, ABPA has been defined as a rare disease in China ${ }^{11}$. Due to ABPA remains under recognized and under diagnosed, it is often misdiagnosed as asthma, tuberculosis, lung cancer and other diseases ${ }^{12-14}$. The typical features of laboratory investigations include increased total IgE level, serum specific IgE levels and peripheral blood eosinophils. In this study, all patients with ABPA fulfill above diagnostic criteria.

CEA is a glycoprotein involved in cell adhesion that is usually produced during fetal development but stops before birth. Serum CEA usually elevated in many cancers including lung, breast, gastric and so on. High levels can also be found in some benign lung conditions. Ahmed Fahim et have demonstrated serum CEA correlates with disease severity in idiopathic pulmonary fibrosis(IPF) ${ }^{15}$. Scholars have found serum CEA elevated in a few ABPA patients ${ }^{16}$. However, there are few studies on the significance of increased CEA levels in ABPA patients. Studies have reported that high CEA levels in serum and bronchoalveolar lavage fluid might connected with mucous embolism in asthma patients ${ }^{17,18}$. Milene Caroline Koch al have also shown that increase CEA levels associated with airway changes in rheumatoid arthritis patients such as bronchial wall thickening, bronchiectasis and nodules ${ }^{19}$. Recent studies have shown that the clinical characteristics and prognosis of ABPA are closely related to the mucus plugs in central bronchiectasis ${ }^{20}$. In accordance with our study in bronchoscopy and imaging manifestations, we found that mucus plugs were seen more frequently in ABPA patients with elevated serum CEA levels, which indicate that serum CEA levels may related with the prognosis of ABPA.

Sensitization to Aspergillus fumigatus is the first step in pathogenesis of ABPA. Repeated exposure to aspergillus fumigatus causes immune response such as IgE elevation, Th2 cytokines high expression, eosinophils infiltration and airway remodeling ${ }^{4}$. Studies have shown that the number of Th2 cells increased in peripheral blood and BALF in patients with ABPA, which reinforce the function of interlukin4(IL-4), further activate $B$ cells and enhance IgE production, which promote disease activity and progression. The increase of IL-5 and GM-CSF will promote eosinophil recruitment, thus exacerbate airway damage ${ }^{21}$. In addition, Khosravi AR et al have found that airway epithelial cells exposed to aspergillus fumigatus spores produced more IFN- $\gamma$, which lead to brief periods of increased IFN- $\gamma$ within the body to promote aspergillus clearance and play a defensive role ${ }^{22,23}$. In our study, ABPA patients with elevated serum CEA levels have a higher level of Th2 cytokines (IL-4 and IL-5),GM-CSF and IFN-y,which indicate that a more intensely inflammatory response occurrence in vivo environment of patients. This reveal that serum CEA levels may related to degree inflammation and disease severity.

Currently, treatment efficacy of ABPA usually evaluated by monitoring pulmonary imaging and serum total IgE levels. There should be an improved radiographic lung changes and a $35 \%$ minimum reduction in total IgE levels ${ }^{24,25}$. Researchers have found that serum CEA levels could be reduced after treatment compared to baseline in ABPA patients ${ }^{16}$. The rare common characteristics of the six cases described in the paper is the extremely high serum CEA level, which decreased after treatment synchronizing with 
serum total IgE levels, suggesting that serum CEA level correlated with the progress in treatment of the disease.

In summary, ABPA patients with elevated CEA levels show a more severe inflammatory response and a poor response to treatment compare to ABPA patients with normal CEA. This indicates that CEA may serve as a monitoring indicator for severity and treatment efficacy of ABPA. Surely, there were limitations in our study. First, we collected blood samples of patients at a single point,and the sample data were incomplete. Second, the numbers of cases we included were relatively small. Our findings were just a hint, in order to better evaluate the immunological characteristics of ABPA and the diagnostic value of serum CEA levels in ABPA, additional studies are required.

\section{Conclusion}

Our study found that CEA may be a predictor of inflammatory condition in patients with allergic bronchopulmonary aspergillosis. This will provide additional meaningful metrics both for clinical and scientific examination.

\section{Abbreviations}

CEA Carcinoembryonic antigen

ABPA allergic bronchopulmonary aspergillosis

CRP C-reactive protein

ESR erythrocyte sedimentation rate

IL-4 interlukin 4

IL-5 interlukin 5

IFN-y interferon-gamma

GM-CSF granulocyte-macrophage colony stimulating factor

\section{Declarations}

\section{Ethics approval and consent to participate}

This work has received approval from the Ethics Committee of Xiangya Hospital Central South University (approval number,201803479), and informed consent was obtained from all participants according to the Declaration of Helsinki.

\section{Consent for publication}


Not applicable.

\section{Contributions}

All authors have contributed to acquisition, analysis of data; writing or editing of the manuscript. All authors read and approved the final manuscript.

\section{Competing interests}

The authors declare no conflicts of interest in this work.

\section{Data Availability Statement}

The datasets generated during and/or analyzed during the current study are available from the corresponding author on reasonable request.

\section{References}

1. Patel G, Greenberger PA. Allergic bronchopulmonary aspergillosis. Allergy Asthma Proc. 2019;40(6):421-424. doi:10.2500/aap.2019.40.4262.

2. Moss RB. Pathophysiology and immunology of allergic bronchopulmonary aspergillosis. Med Mycol. 2005;43(s1):203-6. doi:10.1080/13693780500052255.

3. Mims JW. Asthma: definitions and pathophysiology. Int Forum Allergy Rhinol. 2015;5(Suppl 1):2-6. doi:10.1002/alr.21609.

4. Agarwal R, Chakrabarti A, Shah A, et al. Allergic bronchopulmonary aspergillosis: review of literature and proposal of new diagnostic and classification criteria. Clinical Experimental Allergy. 2013;43(8):850-73. doi:10.1111/cea.12141.

5. Knutsen AP. Lymphocytes in allergic bronchopulmonary aspergillosis. Front Biosci. 2003;8:d589602.

6. Hammarström S. The carcinoembryonic antigen (CEA) family: structures, suggested functions and expression in normal and malignant tissues. Semin Cancer Biol. 1999;9(2):67-81.

7. Nicholson BD, Shinkins B, Pathiraja I, et al. Blood CEA levels for detecting recurrent colorectal cancer. Cochrane Database Syst Rev. 2015;(12):CD011134. doi:10.1002/14651858.CD011134.pub2.

8. Loewenstein MS, Zamcheck N. Carcinoembryonic antigen (CEA) levels in benign gastrointestinal disease states. Cancer. 1978;42(3 Suppl):1412-8.

9. Takehara K, Takehara Y, Ueyama S, Kobayashi T. A case of stercoral colitis with marked elevation of serum carcinoembryonic antigen. Clinical case reports. 2020;8(4):734-8. doi:10.1002/ccr3.2739.

10. Hinson KFW, Moon AJ, Plummer NS. Broncho-pulmonary aspergillosis; a review and a report of eight new cases. Thorax. 1952;7(4):317-33. 
11. Denning DW, Pleuvry A, Cole DC. Global burden of allergic bronchopulmonary aspergillosis with asthma and its complication chronic pulmonary aspergillosis in adults. Med Mycol. 2013;51(4):36170. doi:10.3109/13693786.2012.738312.

12. Patil S, Patil R. "Fleeting pulmonary infiltrates in allergic bronchopulmonary aspergillosis" Misdiagnosed as tuberculosis. International Journal of Mycobacteriology. 2018;7(2):186. doi:10.4103/ijmy.ijmy_57_18.

13. Le Thuong V, Nguyen Ho L, Tran Van N. Allergic Bronchopulmonary Aspergillosis masquerading as recurrent bacterial pneumonia. Med Mycol Case Rep. 2016;12:11-3. doi:10.1016/j.mmcr.2016.06.004.

14. Feng $H$, Lv P, Ren X, Dai H, Yang T. Misinterpretation of allergic bronchopulmonary aspergillosis/allergic bronchopulmonary mycosis due to diverse characteristics in different clinical stages. J Thorac Dis. 2019;11(11):4484-91. doi:10.21037/jtd.2019.10.78.

15. Fahim A, Crooks MG, Wilmot R, Campbell AP, Morice AH, Hart SP. Serum carcinoembryonic antigen correlates with severity of idiopathic pulmonary fibrosis. Respirology. 2012;17(8):1247-52. doi:10.1111/j.1440-1843.2012.02231.x.

16. Noguchi T, Yamamoto K, Moriyama G, et al. Evaluation of serum levels of carcinoembryonic antigen in allergic bronchopulmonary aspergillosis. J Nippon Med Sch. 2013;80(6):404-9.

17. Matsuo K, Araki M, Watanabe $Y$, Hiraki S. [A patient with bronchial asthma and mucoid impaction who presented with a high concentration of carcinoembryonic antigen in serum]. Nihon Kyobu Shikkan Gakkai Zasshi. 1997;35(8):883-7.

18. Maeda Y, Hizawa N, Fukui Y, et al. [Concentrations of carcinoembryonic antigen in serum and bronchoalveolar lavage fluid of asthmatic patients with mucoid impaction]. Nihon Kokyuki Gakkai Zasshi. 2004;42(12):988-93.

19. Koch MC, Pereira IA, Nobre LFS, Neves FS. Computed tomography of pulmonary changes in rheumatoid arthritis: carcinoembryonic antigen (CEA) as a marker of airway disease. Rheumatol Int. 2016;36(4):531-9. doi:10.1007/s00296-016-3438-y.

20. Lu H-W, Mao B, Wei P, et al. The clinical characteristics and prognosis of ABPA are closely related to the mucus plugs in central bronchiectasis. Clin Respir J. 2020;14(2):140-7. doi:10.1111/crj.13111.

21. Chu HW, Wang JM, Boutet M, Boulet LP, Laviolette M. Immunohistochemical detection of GM-CSF, IL4 and IL-5 in a murine model of allergic bronchopulmonary aspergillosis. Clin Exp Allergy. 1996;26(4):461-8.

22. Khosravi AR, Alheidary S, Nikaein D, Asghari N. Aspergillus fumigatus conidia stimulate lung epithelial cells (TC-1 JHU-1) to produce IL-12, IFNy, IL-13 and IL-17 cytokines: Modulatory effect of propolis extract. J Mycol Med. 2018;28(4):594-8. doi:10.1016/j.mycmed.2018.09.006.

23. Shao C, Qu J, He L, et al. Transient overexpression of gamma interferon promotes Aspergillus clearance in invasive pulmonary aspergillosis. Clin Exp Immunol. 2005;142(2):233-41.

24. Agarwal R, Aggarwal AN, Sehgal IS, Dhooria S, Behera D, Chakrabarti A. Utility of IgE (total andAspergillus fumigatusspecific) in monitoring for response and exacerbations in allergic 
bronchopulmonary aspergillosis. Mycoses. 2016;59(1):1-6. doi:10.1111/myc.12423.

25. Patel AR, Patel AR, Singh S, Singh S, Khawaja I. Treating Allergic Bronchopulmonary Aspergillosis: A Review. Cureus. 2019;11(4):e4538. doi:10.7759/cureus.4538.

Figures
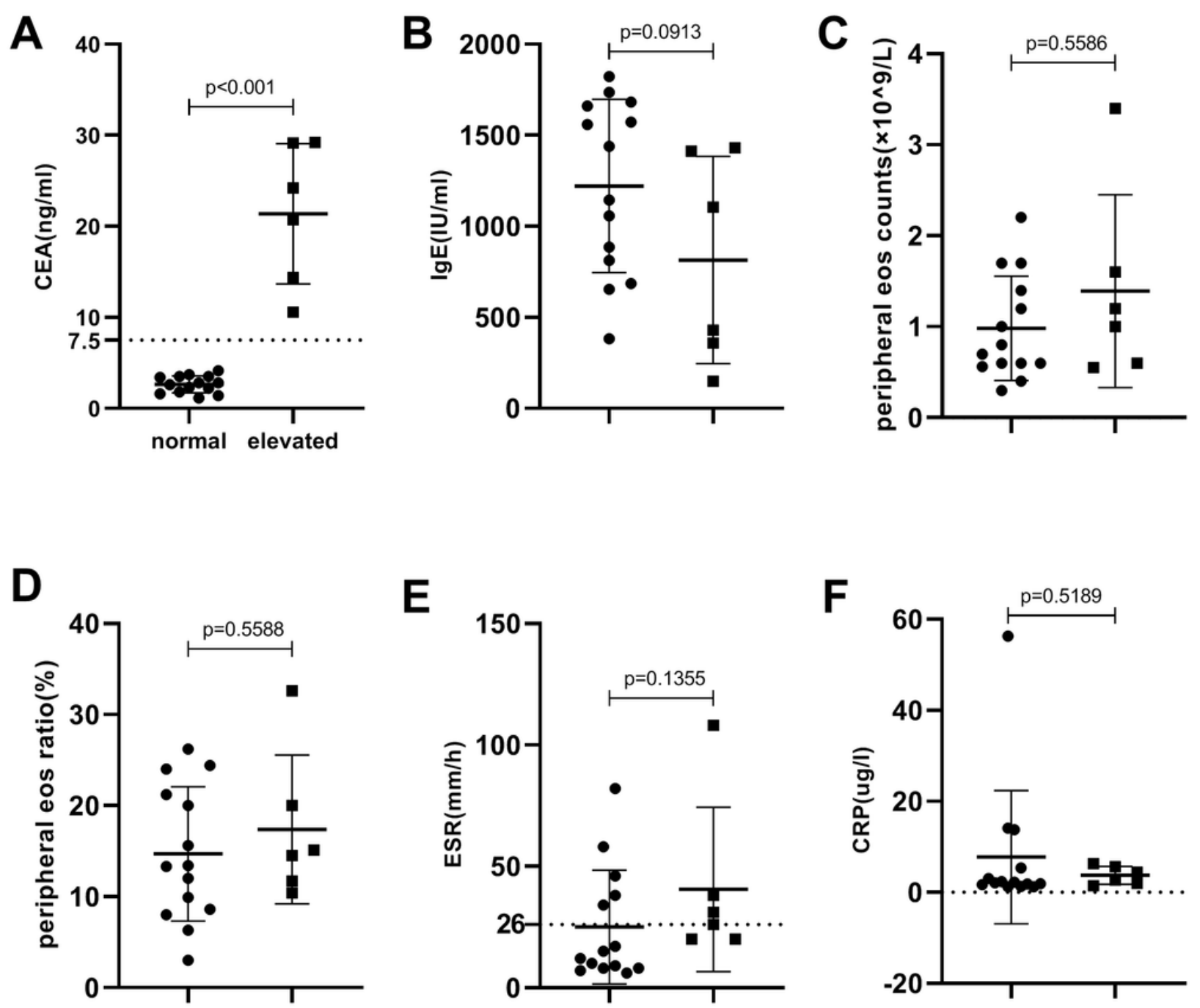

Figure 1

Clinical and immunological data of ABPA patients (two groups: CEA normal $(n=14)$ and CEA elevated $(n=6)$ ). Serum CEA levels $(A)$; serum total IgE levels $(B)$; peripheral eosinophil counts and ratios (C); serum ESR(E) and CRP(F) in ABPA. ABPA allergic bronchopulmonary aspergillosis, CEA Carcinoembryonic antigen. 

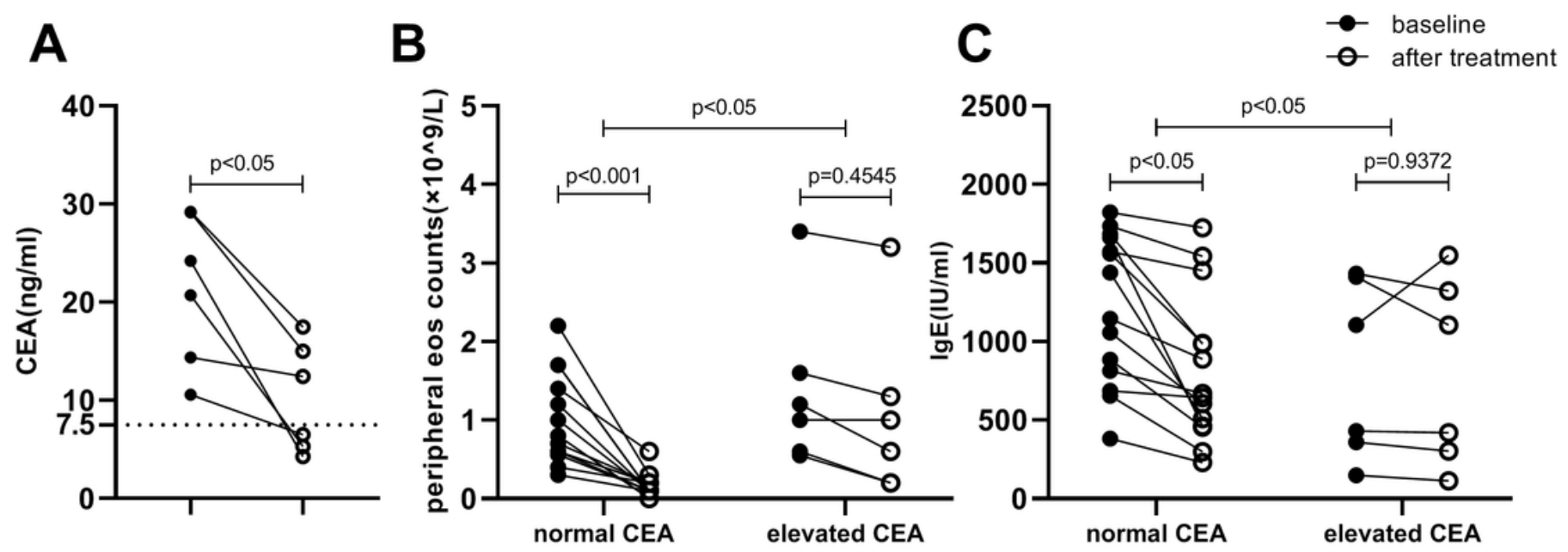

\section{Figure 2}

Longitudinally observation of serum levels of $C E A(A)$, peripheral blood eosinophil counts $(B)$ and serum total IgE levels $(C)$ in patients with ABPA (two groups $\triangle C E A$ normal $(n=14)$ and CEA elevated $(n=6)$ ). ABPA allergic bronchopulmonary aspergillosis, CEA Carcinoembryonic antigen. 

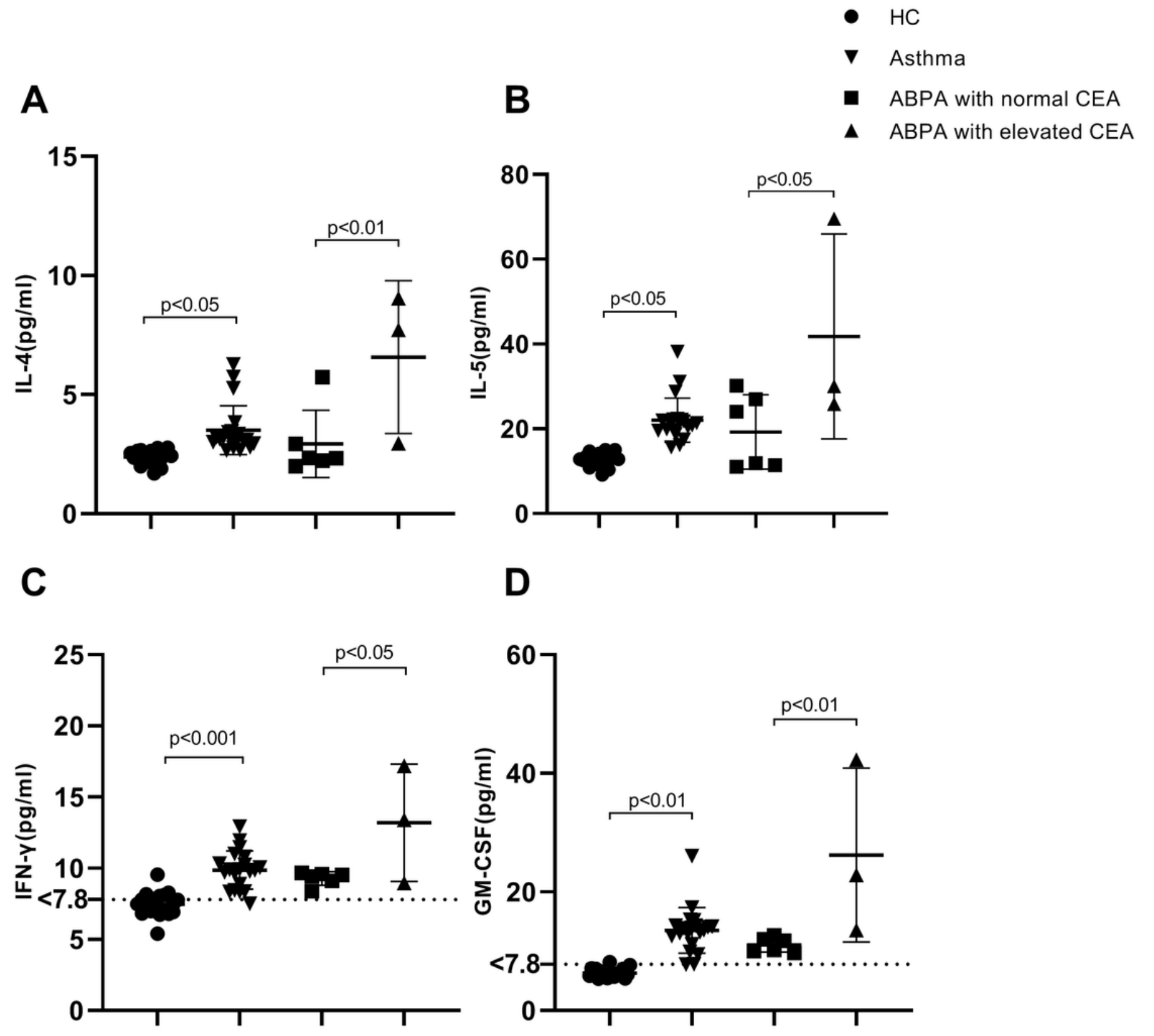

Figure 3

Serum levels of IL-4(A),IL-5 (B),IFN- $\gamma(C)$ and $G M-C S F(D)$ in healthy controls $(n=20)$, patients with asthma $(n=20)$ and ABPA囚two groups: CEA normal $(n=6)$ and CEA elevated $(n=3)$. HC healthy controls, ABPA allergic bronchopulmonary aspergillosis, CEA Carcinoembryonic antigen. 